\title{
TU/e emonownen

\section{Accurate estimation of the time-of-arrival of partial discharge pulses in cable systems in service}

\section{Citation for published version (APA):}

Wagenaars, P., Wouters, P. A. A. F., Wielen, van der, P. C. J. M., \& Steennis, F. (2008). Accurate estimation of the time-of-arrival of partial discharge pulses in cable systems in service. IEEE Transactions on Dielectrics and Electrical Insulation, 15(4), 1190-1199. https://doi.org/10.1109/TDEI.2008.4591242

DOI:

10.1109/TDEI.2008.4591242

Document status and date:

Published: 01/01/2008

\section{Document Version:}

Publisher's PDF, also known as Version of Record (includes final page, issue and volume numbers)

\section{Please check the document version of this publication:}

- A submitted manuscript is the version of the article upon submission and before peer-review. There can be important differences between the submitted version and the official published version of record. People interested in the research are advised to contact the author for the final version of the publication, or visit the $\mathrm{DOI}$ to the publisher's website.

- The final author version and the galley proof are versions of the publication after peer review.

- The final published version features the final layout of the paper including the volume, issue and page numbers.

Link to publication

\section{General rights}

Copyright and moral rights for the publications made accessible in the public portal are retained by the authors and/or other copyright owners and it is a condition of accessing publications that users recognise and abide by the legal requirements associated with these rights.

- Users may download and print one copy of any publication from the public portal for the purpose of private study or research.

- You may not further distribute the material or use it for any profit-making activity or commercial gain

- You may freely distribute the URL identifying the publication in the public portal.

If the publication is distributed under the terms of Article 25fa of the Dutch Copyright Act, indicated by the "Taverne" license above, please follow below link for the End User Agreement:

www.tue.nl/taverne

Take down policy

If you believe that this document breaches copyright please contact us at:

openaccess@tue.nl

providing details and we will investigate your claim. 


\title{
Accurate Estimation of the Time-of-Arrival of Partial Discharge Pulses in Cable Systems in Service
}

\author{
P. Wagenaars, P. A. A. F. Wouters \\ Eindhoven University of Technology \\ Faculty of Electrical Engineering, Electrical Power Systems \\ P.O. Box 513, 5600 MB Eindhoven, The Netherlands \\ P. C. J. M. van der Wielen and E. F. Steennis \\ KEMA \\ P.O. Box 9035, 6800 ET Arnhem, The Netherlands
}

\begin{abstract}
Accurate location of the origins of partial discharges in power cable systems, based on arrival times, is imperative for the identification and assessment of defects. This paper evaluates different time-of-arrival algorithms in order to determine which method yields most accurate location under different circumstances. These methods are based on trigger level, Akaike Information Criterion, energy criterion, Gabor's signal centroid and phase in frequency domain. Several criteria are defined by which the algorithms are evaluated. These criteria include the sensitivity to noise, pulse shape and effect of load impedance. The sensitivity of the methods upon varying these quantities is evaluated analytically and by means of simulations. Further, the methods are tested on a medium-voltage cable system by injecting PD pulses in a cable with one joint. Each algorithm is applied to the measured pulses and the resulting location is compared with the known joint location. From the results the energy criterion method and the phase method show the best performance.
\end{abstract}

Index Terms - Delay estimation, defect location, partial discharges, power cables, signal analysis.

\section{INTRODUCTION}

PARTIAL discharge (PD) diagnostics is a proven method to assess the condition of a power cable system for a condition based maintenance strategy. It is essential to determine the origin of the discharges to estimate the likely defect type and severity. Furthermore, it is only possible to replace parts in a cost-effective manner if the defect locations are known.

The accuracy of defect location depends on the accuracy of the time-of-arrival estimate of each pulse and how accurate the propagation time of the entire cable is known. Nowadays, continuous PD measurements, while the cable remains in service, are also possible (see for example [1-4]). Continuous partial discharge monitoring generates massive data streams and an automated algorithm is required to determine the timeof-arrival of PD pulses with high accuracy. The location methods analyzed in this paper are generally applicable to different PD location systems. However, our main interest concerns online application with its obvious important advantages, but also its inherent additional complications. Ring Main Units (RMUs) or substations distort the pulse shape of both PD signal and injected signal at the cable end.

Manuscript received on 29 November 2007, in final form 13 February 2008.
Furthermore, when a cable remains in service it also is connected to the rest of the grid. Therefore, the measurements are impeded with higher noise and disturbance levels.

PD location in cable systems can be achieved with a single sensor if the far end is disconnected, or with two sensors (one at each cable end) if the cable is in normal operation (see [1]). Both methods need to estimate the total propagation time of the cable $\left(t_{c}\right)$ and the time-of-arrival $\left(t_{o a}\right)$ of PD pulses in order to locate the origins of the PDs. The propagation time $t_{c}$ is determined using the time-of-arrival of injected pulses and their responses.

In this paper five algorithms are evaluated and compared on basis of different criteria. The algorithms are based on:

- Trigger level

- Signal energy

- Akaike Information Criterion (AIC)

- Gabor centroid

- Phase in frequency domain

A set of evaluation criteria, including the sensitivity to noise level and spectrum, the pulse shape, and effect of RMU impedance is used for comparison. The methods are analyzed analytically and by simulations. The results are verified experimentally. 


\section{EVALUATION CRITERIA}

In order to make a well-founded comparison of the strong and weak points of each method a set of evaluation criteria is defined on which the methods are judged. The following criteria are used:

- Arrival time should be independent of noise level and spectrum. The stochastic variation in $t_{o a}$ due to noise should be as small as possible.

- Arrival time should be independent of pulse shape and amplitude.

- Arrival time should be independent of length of recorded signal.

- Arrival time should be independent of the pulse location (in time) in the record.

- Arrival time should be insensitive to reflections of the main pulse on joints and RMUs. These reflections are often present in measured signals.

- Combination of arrival times of injected pulses and measured PD pulses should result in an accurate location of the PD origin.

A $t_{\mathrm{oa}}$-method that meets all these criteria would be the perfect method. But even if not all criteria are met, the method can be useful in specific situations. For PD diagnostics on power cables a location uncertainty of $1 \%$ of the cable length is usually considered to be sufficient.

\section{TIME-OF-ARRIVAL METHODS}

Each method is analyzed analytically with respect to the criteria mentioned in the previous section. Signal, noise and their associated power and energy are defined as follows.

A recorded pulse signal is a combination of the actual pulse signal and additive noise:

$x_{k}=s_{k}+n_{k}$

where $x_{k}$ is the recorded time-discrete signal, $s_{k}$ is the noisefree PD-pulse signal and $n_{k}$ is additive, uncorrelated Gaussian noise with zero mean, $k$ an integer ranging from 1 to $N$ and $N$ the total number of samples in the signal. The signal energy in a record is:

$E_{x}=\sum_{k=1}^{N} x_{k}^{2}=\sum_{k=1}^{N} s_{k}^{2}+\sum_{k=1}^{N} n_{k}^{2}=E_{s}+E_{n}$

with $E_{x}$ the total energy in the recorded signal, $E_{s}$ the energy of the PD signal and $E_{n}$ the noise energy. The mean signal power in a record is:

$P_{x}=\frac{1}{N} E_{x}=\frac{E_{s}}{N}+\frac{E_{n}}{N}=P_{s}+P_{n}$

where $P_{x}$ is the total signal power, $P_{s}$ the PD signal power and $P_{n}$ the noise power. Note that $P_{n}$ is equal to the variance. A PD signal has a finite duration and thus $E_{s}$ is finite if $N \rightarrow \infty$. The noise on the other hand is time-invariant and has constant power $P_{n}$, and thus $E_{n} \rightarrow \infty$ if $N \rightarrow \infty$. Finite-energy disturbances, such as thyristor pulses, are not considered in this paper.

Two of the $t_{o a}$-methods use the noise power $P_{n}$ in their evaluation. The noise power $P_{n}$ can be estimated using a noise measurement prior to the PD measurement, or using a signal of a PD measurement and calculating $P_{n}$ from a part of the signal that contains only noise.

\subsection{TRIGGER LEVEL METHOD}

The trigger level method positions $t_{o a}$ at the time at which the signal $x_{k}$ exceeds a certain threshold level $x_{\text {thres }}$. This is a straightforward method that can easily be implemented [5] and is therefore used in many PD detection systems. The threshold level is chosen relative to the noise level, making it always as low as the noise permits without too many false triggers. First, the algorithm will determine the noise power $P_{n}$ from a signal sample without PD pulse. Next, the threshold is chosen using:

$x_{\text {thres }}=m \cdot \sqrt{P_{n}}$

where $m$ is a parameter chosen by the user. In this paper the value $m=5$ is used for all simulations. In Figure 1a a recorded pulse is plotted together with the threshold $x_{\text {thres }}$.

The $t_{o a}$ determined by this method is noise-level dependent since the threshold $x_{\text {thres }}$ is chosen relative to the noise level. The arrival time of steep pulses is not very sensitive to the noise level, while the arrival time of slow rising pulses can be very sensitive.

\subsection{AKAIKE INFORMATION CRITERION METHOD}

The Akaike Information Criterion (AIC) is a measure of the goodness of fit of a statistical model to a set of observations [6]. It optimizes to a good trade-off between the complexity of the model and the goodness of fit. In seismology this criterion has been used in combination with two autoregressive (AR) fits to determine the onset of seismic waves (see for example [7]). Alternatively, the AIC can be calculated directly from the signal itself [8]. The direct AIC method has been used for ultrasound signals in concrete [9] and PD detection in cable systems [10].

The direct AIC method used in this paper defines an AIC value for each sample $k$ of a signal:

$A I C_{k}=k \cdot \ln \left(\sigma_{1, k}^{2}\right)+(N-k-1) \cdot \ln \left(\sigma_{k+1, N}^{2}\right)$

with $k$ an index ranging from 1 to $N, N$ the total number of samples in signal $x_{k}$ and $\sigma_{m, n}^{2}$ the variance of signal $x_{k}$ from index $m$ up to and including index $n$. The value $A I C_{k}$ is calculated for each sample in the signal $x_{k}$. The $t_{o a}$ is the global minimum of all $A I C_{k}$ values. Figure $1 \mathrm{~b}$ shows a signal and its corresponding AIC curve. 


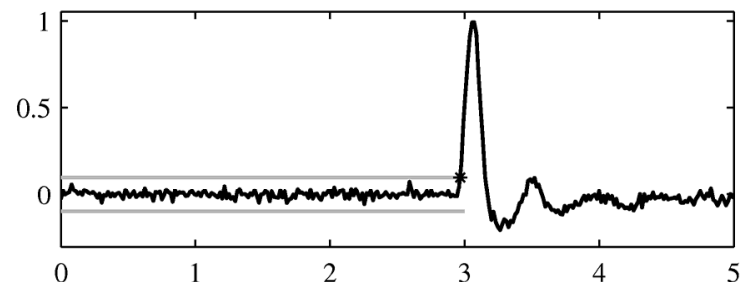

(a) Threshold

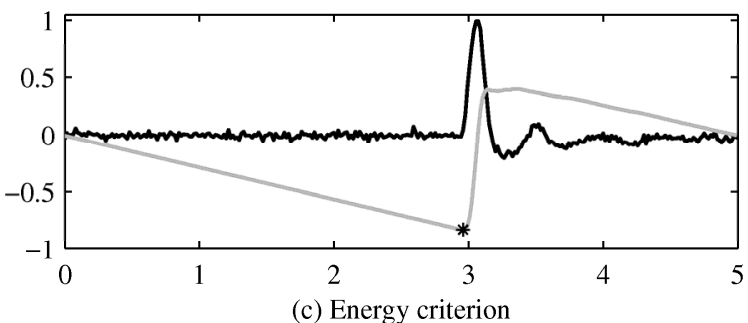

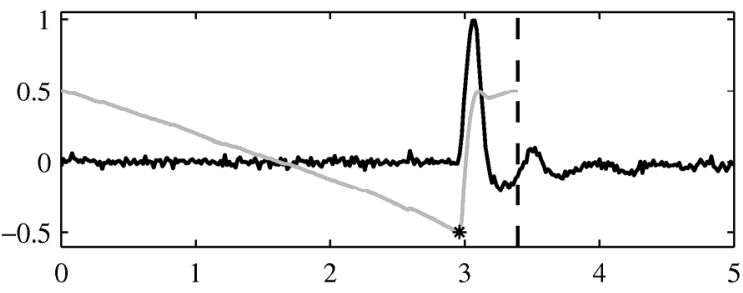

(b) AIC

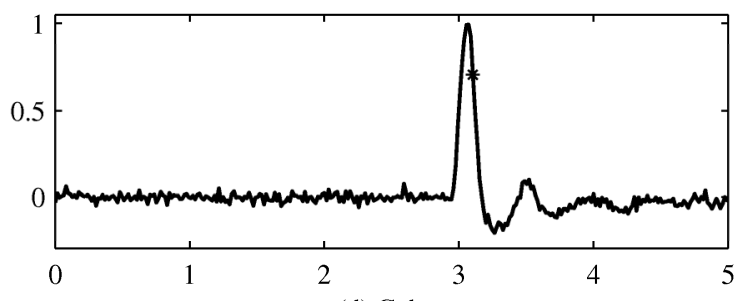

(d) Gabor

Figure 1. Example of recorded pulse with four $t_{o a}$-methods applied. Time axes are in $\mu$ s. Black line: recorded PD signal, gray line: threshold ${ }^{(\text {a) }} /$ normalized $\&$ shifted AIC curve ${ }^{(b)} /$ normalized EC curve ${ }^{(\mathrm{c})}, *$ : time-of-arrival, and dashed line: end of record used for AIC analysis.

Equation (5) is symmetric in the sense that the second term is equal to the first term, except that it starts at the end of the signal. Consider a signal containing a pulse shorter than half of the signal length. Applying this method to the total signal when the pulse is in the first half of the signal and the second half contains only noise, will yield a different $t_{o a}$ (relative to the pulse), than when applied to a similar signal with the same pulse in the second half of the signal. Arranging that the onset of the pulse is always in the second half of the signal, as suggested in [9], avoids this potential pitfall. The tail of the pulse can be cropped at the end of the signal.

At the global minimum of $A I C_{k}$ is $\partial A I C_{k} / \partial k$ equal to zero.

$$
\begin{gathered}
\frac{\partial A I C_{k}}{\partial k}=\ln \left(\sigma_{1, k}^{2}\right)+\frac{k}{\sigma_{1, k}^{2}} \frac{\partial \sigma_{1, k}^{2}}{\partial k}-\ln \left(\sigma_{k+1, N}^{2}\right) \\
+\frac{N-k-1}{\sigma_{k+1, N}^{2}} \frac{\partial \sigma_{k+1, N}^{2}}{\partial k}
\end{gathered}
$$

Since equation (6) contains $\sigma_{l, k}^{2}$, which strongly depends on the noise level, the $t_{o a}$ will be influenced by the noise level. This sensitivity depends on the steepness of the pulse.

\subsection{ENERGY CRITERION METHOD}

The energy criterion (EC) method is based on the energy content of the signal. This method combines the partial signal energy with a negative trend. It has been used for ultrasound signals [9] and PD diagnostics [10]. The signal $E C_{k}$ is defined as:

$$
E C_{k}=E_{x, k}-k \cdot P_{x}
$$

with $E_{k}$ the partial signal energy of signal $x_{k}$ defined as:

$$
E_{x, k}=\sum_{i=1}^{k} x_{i}^{2}
$$

The value $E C_{k}$ is calculated for $k$ ranging for 1 to $N$. The global minimum of all $E C_{k}$ values coincides with the $t_{o a}$. In [8, 9] a factor $\alpha$ is introduced in the second term to move the $t_{o a}$ closer towards the base of the pulse. In this paper this factor is omitted since it introduced a noise-dependency and had no significant influence on the overall location accuracy. Figure 1c depicts a signal, its corresponding EC curve, and the $t_{o a}$.

Substituting $x_{k}=s_{k}+n_{k}$ in equation (7) and assuming the noise to be time-invariant yields:

$E C_{k}=E_{s, k}+E_{n, k}-k \cdot P_{s}-k \cdot P_{n}=E_{s, k}-k \cdot P_{s}$

At the global minimum in equation (9):

$\frac{\partial E C_{k}}{\partial k}=0=s_{k}^{2}-P_{s}$

The noise $n_{k}$ is not present in equation (10), indicating that $t_{o a}$ is independent of the noise level. Since a PD pulse has a finite duration, the mean signal power $P_{s}$ depends on the length of the signal. As a consequence $t_{o a}$ depends on the signal length. All analyses are done using the same record length to prevent a record-length dependency in the results.

\subsection{GABOR CENTROID METHOD}

Gabor defines the "epoch" of order 1 of a signal [11]. Assuming that the signal is real, converting it to time-discrete form and substituting $x_{k}=s_{k}+n_{k}$ this epoch is defined as:

$t_{g}=\frac{\sum_{k=1}^{N} t_{k} x_{k}^{2}}{\sum_{k=1}^{N} x_{k}^{2}}=\frac{\sum_{k=1}^{N} t_{k} s_{k}^{2}+P_{n} \sum_{k=1}^{N} t_{k}}{\sum_{k=1}^{N} s_{k}^{2}+P_{n} \cdot N}$

where $t_{g}$ is Gabor's epoch and $t_{k}$ the time corresponding to sample $k$. This equation shows that $t_{g}$ is dependent on the noise 
level. This error can be corrected using:

$$
t_{o a, g}=\frac{\sum_{k=1}^{N} t_{k} x_{k}^{2}-P_{n} \sum_{k=1}^{N} t_{k}}{\sum_{k=1}^{N} x_{k}^{2}-P_{n} \cdot N}
$$

The noise power $P_{n}$ can be estimated based on a part of signal $x_{k}$ that has guaranteed no pulse, or on a separate noise measurement. Figure 1d shows a signal and its corresponding $t_{\text {oa, } \mathrm{g}}$.

\subsection{PHASE METHOD}

The phase method converts the recorded signal $x_{k}$ to frequency domain and retrieves the phase for a chosen frequency. A time delay is represented in frequency domain as:

$$
X_{\text {delayed }}(\omega)=X(\omega) \cdot e^{-j \omega \tau}
$$

where $\tau$ is the delay time and $X_{\text {delayed }}(\omega)$ is $X(\omega)$ delayed for $\tau$. The phase method uses this relation and defines the arrival time as:

$$
\tau=-\frac{\angle X\left(\omega_{c}\right)}{\omega_{c}}
$$

where $X(\omega)$ is the recorded signal converted to frequency domain, $\angle$ denotes the phase of a signal in frequency domain and $\omega_{c}$ is a chosen frequency. For all pulses the same $\omega_{c}$ must be used.

Due to the periodicity of $2 \pi$ the phase is not unambiguously determined by equation (14):

$$
\tau=\frac{-\angle X\left(\omega_{c}\right)}{\omega_{c}}+\frac{m \cdot 2 \pi}{\omega_{c}}
$$

with $m$ an unknown integer. This problem can be solved by multiplying $X(\omega)$ with a (negative) time delay $\tau_{c h}$ (see equation (13)) first. This time delay must be chosen such that the phase does no longer wrap around (jump from $-\pi$ to $\pi$, or vice versa). Since $\tau_{c h}$ is unknown a priori, an iteration loop is used to find the correct time delay. Once the correct $\tau_{c h}$ has been found the phase of the delayed $X(\omega)$ is converted to a time using equation (14) and added to $\tau_{c h}$. The entire procedure is implemented using the following steps:

1) Convert recorded signal $x_{k}$ to frequency domain $X(\omega)$.

2) Determine frequency range(s) over which $X(\omega)$ has a good signal-to-noise ratio. In these ranges the phase of $X(\omega)$ is most reliable.

3) Start iteration loop.

4) Add time delay $\tau_{c h}$ (an initial value or a value based on previous iteration step) to $X(\omega)$ :

$$
X_{c h}(\omega)=X(\omega) \cdot e^{j \omega \tau} \tau_{c h}
$$

5) Check the phase of $X_{c h}(\omega)$ for phase jumps of $\pm 2 \pi$ in the frequency ranges determined in step 2 . If there are jumps, go back to step 3 and choose a new $\tau_{c h}$. If not, proceed with the next step.

6) Calculate the time-of-arrival using:

$$
t_{o a, p}=\tau_{c h}-\frac{\angle X_{c h}\left(\omega_{c}\right)}{\omega_{c}}
$$

where $\omega_{c}$ is a frequency within the range determined in step 2. For consistency, $\omega_{c}$ must be the same for all measurements. Taking the average over a small frequency range around $\omega_{c}$ reduces the sensitivity to noise and reflections in the signal. In this paper $\omega_{c}=2 \pi \cdot 1.5 \mathrm{MHz}$ is used together with a range of $\pm 500 \mathrm{kHz}$ for averaging to reduce noise sensitivity.

In Figure 2 a recorded PD signal is plotted in time domain together with the phase of the signal in frequency domain. After the iteration loop (steps 3-5) the signal is shifted in time domain towards $t=0$. This shifted signal is also plotted in Figure 2. Due to the nature of the discrete Fourier transform the first part of the pulse is wrapped to the end of the signal, which is not shown.

The major advantage of this method is that it is not influenced by dispersion and attenuation of the cable. In the absence of complications due to a load impedance $\left(Z_{\text {load }}\right)$ the mean location error will be zero. Choosing a narrow but finite frequency range around $\omega_{c}$ introduced only an insignificant error. Unfortunately, for online diagnostics, signal transmission to the load impedances at the RMUs introduces a phase shift. If the characteristic cable impedance $\left(Z_{c}\right)$ and the
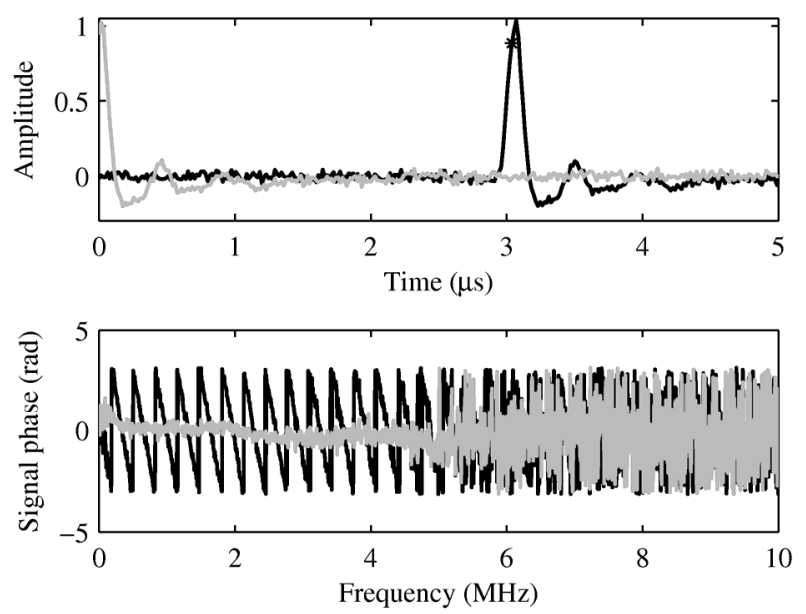

Figure 2. Signal before and after iteration loop. The signal is plotted in time domain and the phase in frequency domain. The iteration loop ended with $\tau_{c h}=3.05 \mu \mathrm{s}$. Black line: signal before iteration, gray line: signal after iteration loop, and *: time-of-arrival. 
load impedance are known, e.g. by using an impedance measurement as described in [13], the phase shift can be estimated. This estimate can be used to corrected $t_{o a, p}$ :

$t_{o a, p, c o r r}=t_{o a, p}+\frac{\angle T\left(\omega_{c}\right)}{\omega_{c}}$

where $t_{\text {oa,p,corr }}$ is the corrected arrival time and $T$ is the current transmission coefficient from cable to load, given by $T(\omega)=2 Z_{c} /\left(Z_{c}+Z_{\text {load }}\right)$. However, the impedance estimation has limited accuracy depending on the RMU configuration. In the appendix the sensitivity of the location accuracy to errors in the impedance estimation is analyzed.

Since there is no correlation between the phase of the signal at $\omega_{c}$ and the phase of the noise, the noise has no influence on the expected value of $t_{o a, p}$, but only on it's standard deviation.

\section{PD LOCATION}

For the location of a PD two measurements are required: a cable propagation time $\left(t_{c}\right)$ measurement and the actual PD measurement. Offline diagnostics often uses a single-sided system where the propagation time measurement and PD location are based on reflections from the far end [12]. The impedances of RMUs connected to the cable during online measurements usually prevent visible reflections from PD signals. Detection of the pulses at both ends is then preferred [13]. Although the analyses are based on a two-sided system, the conclusions of the $t_{o a}$-method evaluation are valid for other PD-measurement configurations.

\subsection{CABLE PROPAGATION TIME MEASUREMENT}

The $t_{c}$-measurement using two, non-synchronized measurement systems (one at each cable end) is conducted as follows. A pulse is injected by measurement unit 1 (MU1) at the near cable end $\left(s_{11}\right)$, which is detected by MU2 at the far end $\left(s_{21}\right)$. After a short delay ( $t_{\text {wait }}$, for example $\left.10 \mu \mathrm{s}\right) \mathrm{MU} 2$ injects a similar pulse $\left(s_{22}\right)$, which is detected by MU1 $\left(s_{12}\right)$. The record taken by each unit must contain both pulses. Figure 3 illustrates schematically the signals recorded by MU1 and MU2.

In the $t_{c}$-measurement there are in total four pulses. The arrival time of each pulse is determined using a $t_{o a}$-method. The time difference between the two pulses recorded by MU1 is given by $\tau_{1}=2 t_{c}+t_{\text {wait }}$, and the between the pulses of MU2 by $\tau_{2}=t_{\text {wait }}$. The cable propagation time $t_{c}$ is equal to: $t_{c}=1 / 2\left(\tau_{1}-\tau_{2}\right)$. The advantage of this method is that the time bases of MU1 and MU2 do not need to be synchronized for the cable propagation time measurement.

\subsection{PD MEASUREMENT}

During the PD measurement the measurement units at each cable end detects a PD pulse. The time bases of the units must be accurately synchronized (for example using pulse injection [13] or GPS). The exact arrival time of the PD pulses is determined using one of the $t_{o a}$-methods. The difference in

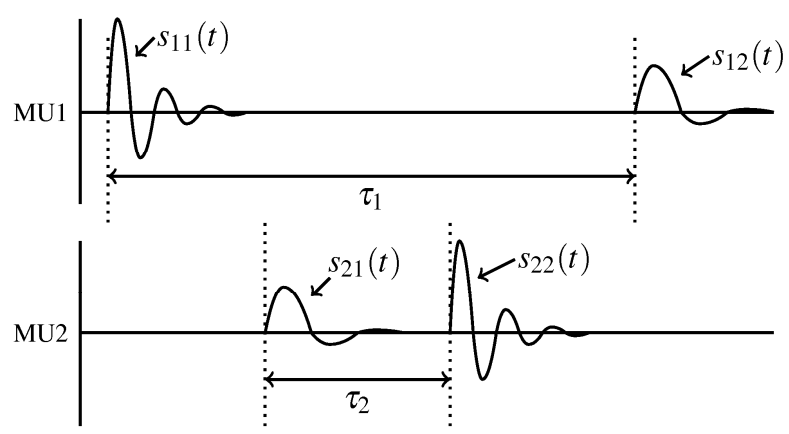

Figure 3. Signals arriving at both cable ends during a propagation time measurement.

time-of-arrival at both ends is used, together with $t_{c}$, to calculate the PD origin:

$$
z_{P D}=\frac{1}{2} l_{c}\left(1-\frac{t_{o a 2}-t_{o a 1}}{t_{c}}\right)
$$

where $z_{P D}$ is the distance from near cable end to the PD origin, $l_{c}$ the cable length and $t_{\text {oal }}, t_{\text {oaz }}$ the arrival times of the PD pulse at respectively MU1 and MU2.

\section{SIMULATIONS}

Several simulations have been conducted to evaluate the $t_{o a^{-}}$ methods using the criteria proposed in section 2. For all simulations a model for a cable of $1 \mathrm{~km}$ is used. The characteristic impedance and propagation coefficient for this cable were measured on a field-aged 3-core MV PILC cable of $200 \mathrm{~m}$ to obtain realistic values in the simulations. At each end the cable is terminated with a load impedance $\left(Z_{\text {load }}\right)$, representing the effect of an RMU or substation, where the sensor is installed. The sensor is assumed to be an ideal current probe that measures the current through the load impedance. The choice for a current probe as sensor is related to the online application in which case usually it is the only sensor type allowed to be installed. Moreover, it is also the most challenging situation because the RMU-cable injection circuit will have a profound effect on the waveforms. The transmission coefficient from cable to load is calculated using the load impedance and the characteristic cable impedance. The load impedance can be varied to investigate the effect on the $t_{o a}$-methods. In the other simulations the load impedance is matched to the cable impedance (real-valued, frequencyindependent impedance of $12 \Omega$ ).

The $t_{c}$-measurement is simulated using different pulse shapes for the injected pulse. Since PDs are short phenomena with respect to $1 / f_{d}\left(f_{d}\right.$ detection bandwidth) a PD signal is represented by a $\delta$-pulse at the origin. All pulses in the simulated signal are normalized to a maximum amplitude of one, so that the signal-to-noise ratio is about the same for all pulses. Uncorrelated Gaussian noise with a specified spectrum is added to the signal to simulate realistic noise conditions. Unless stated otherwise, the noise spectrum taken is white.

Each simulation is repeated 1000 times. The mean and standard deviation of those 1000 repetitions give a good 
estimate for the accuracy and precision of the methods. Due to the stochastic nature of noise the application of the $t_{o a^{-}}$ methods to a simulation returns occasionally an obviously incorrect value, especially with higher noise levels. These outliers are filtered out using the modified z-score method [14], which is based on the median deviation from the median of all 1000 repetitions of the simulation.

\subsection{SENSITIVITY TO NOISE LEVEL}

The accuracy should be independent of the noise level. A $t_{c^{-}}$ measurement using PD-like pulses is simulated at different noise levels. The results are plotted in Figure 4.

The mean values of the EC, Gabor and phase method are virtually independent of the noise level. The threshold and AIC method show a small noise-level dependency. The standard deviation of the Gabor method shows a quadratic relation with the noise level. For higher noise levels the standard deviation of the Gabor method is unacceptably high. The standard deviations of the other methods are within $0.5 \%$ of the mean $t_{c}$.

The methods estimate different cable propagation times. This is related to the distinct backgrounds of the methods. They yield different $t_{o a}$ 's on the same pulse, as shown in Figure 1 and 2. Since there exists no unambiguous definition for $t_{c}$ the accuracy of the methods can not be judged solely on the propagation time. The differences cancel out when the $t_{o a}$ 's are combined to obtain the PD origin. Therefore, the accuracy of the methods can only be tested using the PD location as tested in section 5.4 .

\subsection{SENSITIVITY TO NOISE SPECTRUM}

During a measurement the noise is rarely white. In this section a $t_{c}$-measurement is simulated using three different
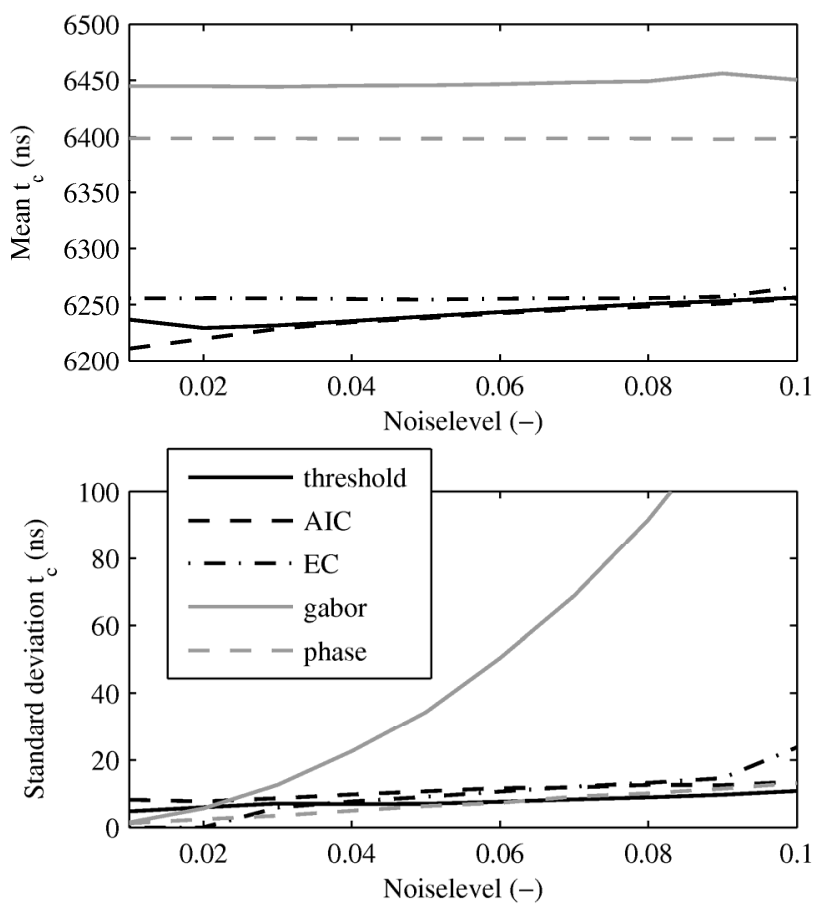

Figure 4. Mean and standard deviation of cable propagation time simulation. Unit of noise level is the ratio of the standard deviation of noise relative to maximum amplitude of pulses. noise conditions shown in Figure 5. In all three situations the noise is Gaussian and has the same mean power. The only difference between the simulations is the noise spectrum. Spectrum 1 is white; spectrum 2 and 3 are computer-generated with a shape similar to spectra measured during field measurements. The difference between 2 and 3 is that spectrum 2 lacks narrow band sources.

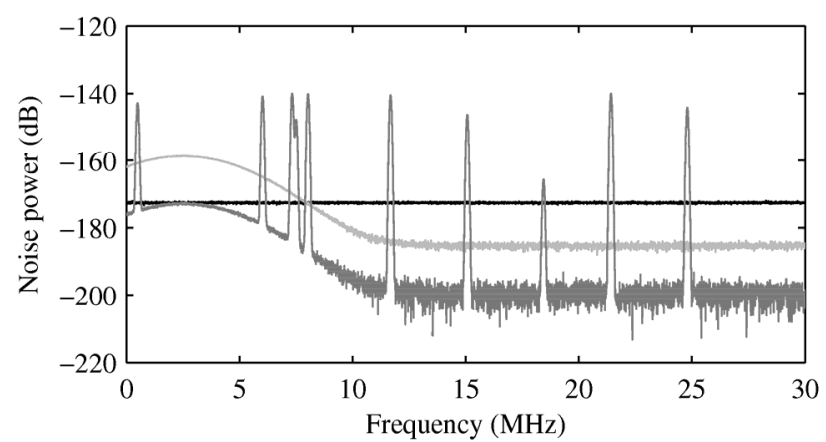

Figure 5. Noise spectra used in the noise-spectrum-sensitivity simulations. Black: situation 1, light gray: situation 2 and dark gray: situation 3.

The mean and standard deviation of the 1000 repetitions of the simulations are given in Table 1. These results show that the $t_{o a}$-methods are virtually insensitive to the noise spectrum. Only the standard deviation of the Gabor method shows a significant change.

\subsection{SENSITIVITY TO PULSE SHAPE}

A $t_{c}$-measurement is performed using pulses injected by the measurement system. There are different methods to couple a pulse into the cable system (capacitive, inductive or galvanic). Different coupling mechanism will inject different pulse shapes. Also, the design of the pulse source and the load impedance where the pulse is injected influence the pulse shape. The cable propagation time $t_{c}$ is independent of the pulse shape. Therefore, a $t_{o a}$-method should yield the same $t_{c}$ for different pulse shapes.

Figure 6 shows three different pulse shapes of injected pulses. Pulse 1 is an inductively coupled pulse that was measured during an online PD measurement in an MV cable system. Most energy of this pulse is concentrated in the beginning of the pulse. The second pulse is also an inductively coupled pulse, but with different pulse source design, different injection coil and different load impedance. This pulse starts with a long low bump (charging) and then a short steep pulse. Thus, most low frequency content is located in the first part of the pulse and most high frequency content comes about $1 \mu \mathrm{s}$ later. This kind of waveforms can arise not only due to the pulse source design as is the case with this pulse, but also due to various signal propagation modes, e.g. in belted cables [15]. The third pulse is a short pulse with a width of about $100 \mathrm{~ns}$.

Table 1. Mean and standard deviation of $t_{c}$ of the noise-spectrum-sensitivity simulations. All values are in ns.

\begin{tabular}{c|ccccc}
\hline Spec. & Threshold & AIC & EC & Gabor & Phase \\
\hline 1 & $6232 \pm 7$ & $6228 \pm 9$ & $6256 \pm 5$ & $6445 \pm 13$ & $6399 \pm 4$ \\
2 & $6231 \pm 7$ & $6229 \pm 10$ & $6256 \pm 6$ & $6445 \pm 21$ & $6399 \pm 8$ \\
3 & $6232 \pm 7$ & $6228 \pm 9$ & $6256 \pm 6$ & $6445 \pm 32$ & $6399 \pm 4$ \\
\hline
\end{tabular}



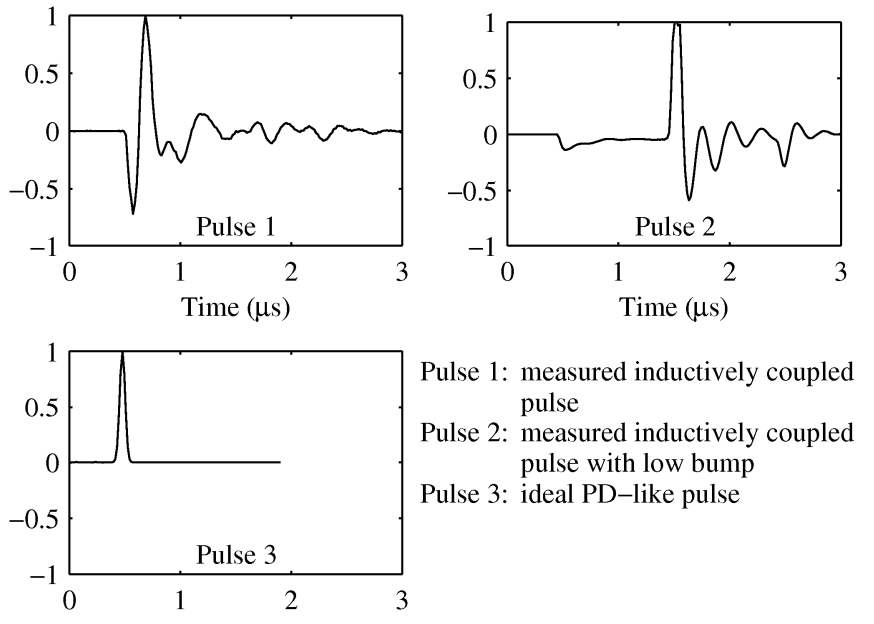

Figure 6. Pulse shapes used in injection in pulse-shape-sensitivity simulations.

This pulse is similar to a real PD pulse that has propagated through a power cable over a short distance.

For each pulse 1000 simulations of $t_{c}$-measurements have been conducted. The mean and standard deviation of each simulation set is summarized in Table 2. Except for the phase method, all methods are sensitive to the pulse shape. Especially pulse 2 yields unacceptable results. There, most high and low frequency content of the pulse are concentrated at different times. At injection the start of the high-frequency part is chosen as time-of-arrival, because it contains most energy. After propagating through the cable most highfrequency content is attenuated, and the low-frequency becomes dominant. Therefore, at the far end the time-ofarrival is determined by the low-frequency part. The only method that is not influenced by the pulse shape is the phase method, because it analyzes the pulse in frequency domain and uses a fixed frequency for all analyses. The change in $t_{c}$ of the Gabor method less than $2 \%$, which is twice the target accuracy of $1 \%$. The differences of the other methods are unacceptable.

Table 2. Mean and standard deviation of $t_{c}$ of the pulse-shape-sensitivity simulations. All values are in ns.

\begin{tabular}{c|ccccc}
\hline Pulse & Threshold & AIC & EC & Gabor & Phase \\
\hline 1 & $6230 \pm 6$ & $6209 \pm 8$ & $6241 \pm 3$ & $6355 \pm 7$ & $6399 \pm 5$ \\
2 & $5333 \pm 10$ & $5272 \pm 12$ & $5391 \pm 10$ & $6318 \pm 11$ & $6399 \pm 3$ \\
3 & $6231 \pm 7$ & $6228 \pm 8$ & $6256 \pm 6$ & $6445 \pm 12$ & $6399 \pm 4$ \\
\hline
\end{tabular}

An option to reduce the pulse shape sensitivity is to calculate the transfer function from the near end pulse to the far end pulse. Converting a transfer function in frequency domain to time domain gives the impulse response of the channel. It can be regarded as the far-end pulse from an injected $\delta$-pulse. The arrival time of the impulse response is $t_{c}$. The calculated transfer functions are:

$H_{12}(\omega)=\frac{S_{21}(\omega)}{S_{11}(\omega)} \quad$ and $\quad H_{21}(\omega)=\frac{S_{12}(\omega)}{S_{22}(\omega)}$

with $H_{12}(\omega)$ the transfer function from near end to far end, and $S_{i j}(\omega)$ the time domain pulse $s_{i j}(t)$ (see Figure 3 ) converted to frequency domain. If time bases of the near and far end are not synchronized the average of the arrival times of $h_{12}(t)$ and $h_{21}(t)$ is equal to the propagation time.

The simulations of the $t_{c}$-measurements with different pulse shapes have been repeated. The $t_{o a}$ is now determined using the channel's impulse response. The results are listed in Table 3. The results for pulse 1 are clearly unusable for all methods, except for the phase method. The results for pulse 2 and 3 are satisfactory. The reason that the results for pulse 1 are far off is the lack of low frequency content. Therefore, the constructed transfer function becomes unreliable at low frequencies and the impulse response is impeded by noise.

Table 3. Mean and standard deviation of $t_{c}$ of the pulse-shape-sensitivity simulations using the channel's impulse response. All values are in ns.

\begin{tabular}{c|ccccc}
\hline Pulse & Threshold & AIC & EC & Gabor & Phase \\
\hline 1 & $3442 \pm$ & $5321 \pm$ & $5896 \pm$ & $6521 \pm$ & $6398 \pm 2$ \\
& 1653 & 688 & 135 & 138 & \\
2 & $6190 \pm 5$ & $6153 \pm 9$ & $6192 \pm 0$ & $6451 \pm 9$ & $6399 \pm 1$ \\
3 & $6183 \pm 6$ & $6143 \pm 6$ & $6192 \pm 0$ & $6446 \pm 4$ & $6399 \pm 1$ \\
\hline
\end{tabular}

\subsection{SENSITIVITY TO LOAD IMPEDANCE}

This section deals with the location accuracy in general and the influence of the load impedance on the accuracy. In order to be able to locate PDs both a $t_{c}$-measurement simulation and the actual PD measurement simulation are required. Since the load impedance at each cable end has a significant influence on the pulse shape the location simulations are conducted with different load impedances. Four different impedances are used: a real impedance matching the cable impedance, a capacitive load, an inductive load and a load impedance measured online in a ring-main-unit (RMU) (see [13] for the online impedance measurement method). The last impedance is a typical load impedance encountered in a medium-voltage distribution grid. The $t_{c}$-measurement was simulated using the short PD-like pulse. The noise is white and has a constant level for all simulations.

In the first simulation the load impedance at both cable ends is equal to the characteristic cable impedance. Therefore the pulse shape will not be distorted at the transition from cable to load impedance. This simulates the location accuracy of the $t_{o a}$-methods when a PD pulse shape is influenced by the dispersion and attenuation of the cable. The mean and standard deviation of the location error of the simulations are plotted in Figure 7. This figure shows that all methods provide an accurate location $(<0.1 \%$ of cable length). Note that the location error of the phase method is virtually zero. The standard deviation of the Gabor method is larger than the other methods, which are similar to each other.

In the second simulation the near end is terminated with an inductance of $1 \mu \mathrm{H}$ and the far end is terminated with a capacitance of $2 \mathrm{nF}$. These impedances do not represent field conditions, but are meant to test the methods for different loads with opposite phase shift at both ends. This is a worstcase scenario for the phase method because the errors introduced due to the phase shift at the transitions to the load impedances at both ends accumulate. The results of the 

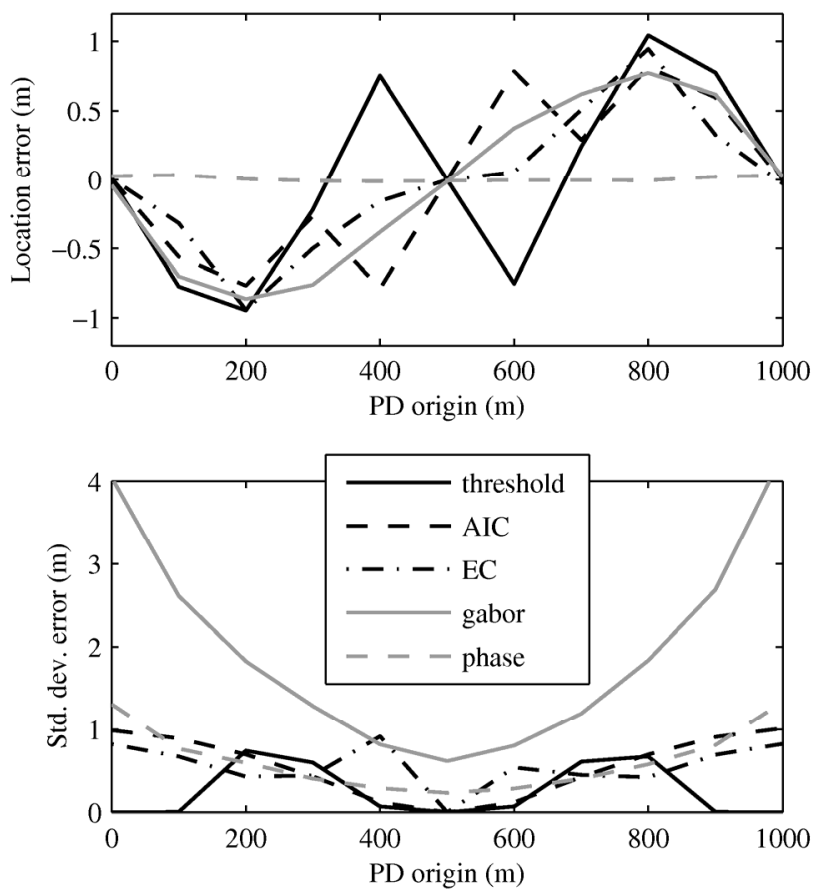

Figure 7. Mean and standard deviation of the location error for PDs from different locations in the cable. Load impedances at both cable ends are matched to the characteristic impedance of the cable.

simulations are plotted in Figure 8. As expected the phase method performs poorly for this configuration. But if the load impedance at the test frequency $\omega_{c}$ would be known, the phase shift at the transmission to the load impedance can be corrected. The performance of the Gabor method is not within the $1 \%$ target accuracy limit and has a relatively large standard deviation. The other three methods have a mean error less than $0.5 \%$ of the cable length.

The third simulation consists of a matched impedance at the near end and at the far end a realistic load impedance that was actually measured in the field. The results are depicted in Figure 9. The AIC method has both the largest mean and standard deviation. The phase method without correction and Gabor method have both a maximum mean error of approximately $10 \mathrm{~m}$ ( $1 \%$ of the cable length). The energy criterion method has both a low mean error and a low standard deviation.

\section{EXPERIMENT}

In order to test the methods experimentally measurements on a test setup have been conducted. The test setup consists of two RMUs with MV/LV transformer and three-core MV Paper Insulated Lead Covered (PILC) cables. A schematic drawing of the test setup is depicted in Figure 10. The MV cable between RMU1 and RMU2 consists of two different field-aged cable sections. The propagation velocity of the first section $(96 \mathrm{~m})$ is about $5 \%$ higher than the other section $(201 \mathrm{~m})$. RMU1 has a second MV cable that can be disconnected. RMU1 has an MV/LV transformer of 1 MVA, and RMU2 has a transformer of $100 \mathrm{kVA}$. The joint can be opened to inject PD-like pulses with a pulse source. Current probes and injection coils are installed in both RMUs as indicated in Figure 10 (see [13] for details on the installation options).
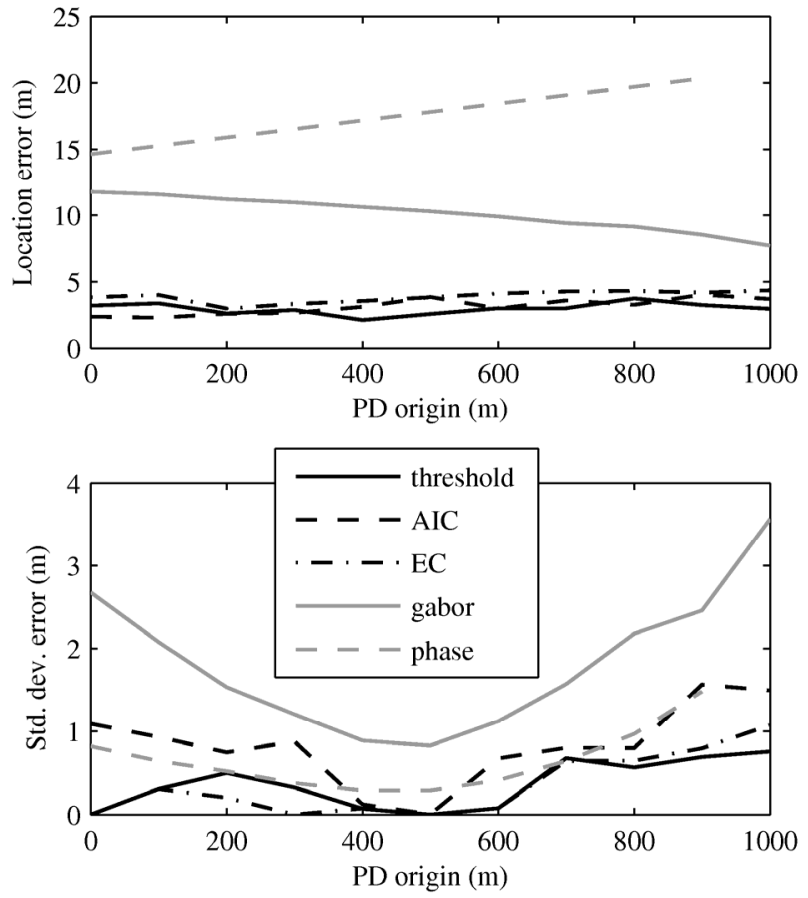

Figure 8. Mean and standard deviation of the location error for PDs from different locations in the cable. Load impedance at near end is and inductance of $1 \mu \mathrm{H}$ and the load impedance at the far end is a capacitance of $2 \mathrm{nF}$.

Two configurations are tested. In the first experiment the parallel cable in RMU1 is disconnected. In both RMUs the load impedance is formed by the transformer and the cables to the transformer. In the second experiment the parallel cable in RMU1 is connected to the rail. The load impedance is now the transformer impedance together with the impedance of the parallel cable. For both measurements first a $t_{c}$-measurement is conducted, followed by a PD measurement where PD-like pulses
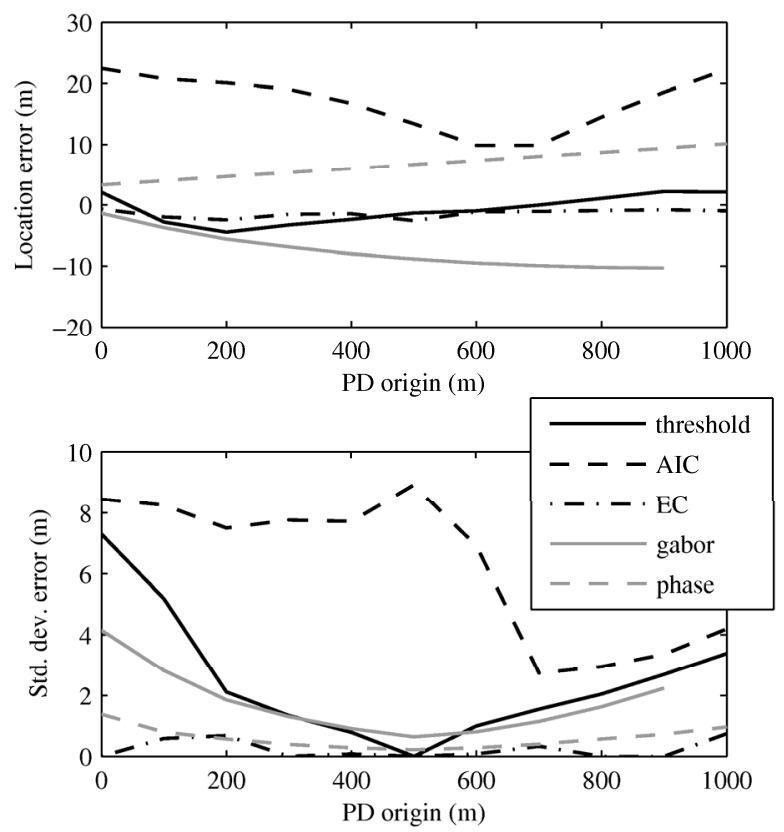

Figure 9. Mean and standard deviation of the location error for location of PDs from different locations in the cable. Load impedance at near end is matched to the cable and load impedance at far end is the load impedance measured in an RMU. 


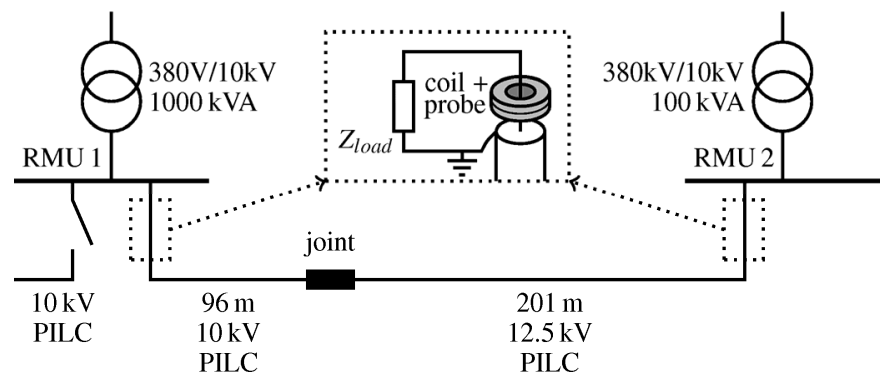

Figure 10. Schematic drawing of test setup. The insert shows the position of the current probe and injection coil in the RMU

are injected in the joint. All measurements are repeated 100 times.

The results of both experiments are listed in Table 4. Equation (19) yields a distance of $93 \mathrm{~m}$ instead of $96 \mathrm{~m}$ due to the difference in propagation speed in the $96 \mathrm{~m}$ section and the $201 \mathrm{~m}$ section. The different propagation velocities can be incorporated in the analysis (see [13]), but for convenience we assume the joint to be at $93 \mathrm{~m}$ and both cable segments to have equal propagation velocities.

The threshold, AIC and EC methods have an accuracy better than $1 \%$ of the cable length in both experiments. The accuracy of the Gabor and (uncorrected) phase methods is insufficient. The accuracy of the phase method can be improved significantly if the load impedances are known around the test frequency $\omega_{c}$, but the analysis in Table 4 does not take this into account. It intends to simulate the worst-case scenario in which this measurement is not performed accurately or not done at all. During experiment 1 the accuracy of the phase method is reasonable, but during the second experiment the accuracy is definitely insufficient. During the first experiment the load impedances in RMU1 and RMU2 were similar, so the phase shifts cancel, while during experiment 2 the load impedance of RMU1 is changed significantly due to the parallel MV cable. A second cause for the relatively large error is that the cable in the experiment is shorter than in the simulations. Therefore, any phase shift at the cable ends introduces in the experiment results a relatively larger error than in the simulations. The inaccuracy of the Gabor method is probably caused by reflections present in the signal. The term $t_{k} x_{k}$ in equation (12) causes reflections coming after the main pulses to have a significant effect on the $t_{o a, g}$.

\section{CONCLUSION}

The $t_{o a}$-methods discussed in this paper have been evaluated analytically and with simulations to investigate the strong and weak points of the methods. As far as practically possible these have been verified experimentally. The strong and weak points are summarized in Table 5.

Altogether, no single method performs superior on all criteria. Depending on the situation either the EC method or the phase method will provide the most reliable overall performance. The EC method has good accuracy in most situations. The only point where it failed is on its sensitivity to particular pulse shapes. Incorporating the channel's impulse response improved the pulse shape sensitivity for some pulse shapes. The strongest point of the phase method is its complete insensitivity to the pulse shape. The second advantage is the high accuracy, provided that the load impedance
Table 4. Mean and standard deviation of location of experiments. All values are in $\mathrm{m}$.

\begin{tabular}{l|ccccc}
\hline & Threshold & AIC & EC & Gabor & Phase \\
\hline Exp. 1 & $93.3 \pm 0.8$ & $93.5 \pm 0.6$ & $94.4 \pm 0.6$ & $87.3 \pm 0.3$ & $96.7 \pm 0.3$ \\
Exp. 2 & $94.3 \pm 0.7$ & $94.1 \pm 0.9$ & $93.6 \pm 0.3$ & $121.9 \pm 0.4$ & $82.7 \pm 0.5$ \\
\hline
\end{tabular}

Table 5. Summary of strong and weak points of $t_{o a}$-methods. See section 2 for a description of the criteria.

\begin{tabular}{c|ccccc}
\hline & Threshold & AIC & EC & Gabor & Phase \\
\hline Noise & - & - & + & 0 & + \\
Pulse shape & $-/ 0^{\mathrm{a}}$ & $-/ 0^{\mathrm{a}}$ & $-/ 0^{\mathrm{a}}$ & $-/ 0^{\mathrm{a}}$ & $+/+^{\mathrm{a}}$ \\
Record length & + & + & 0 & + & + \\
$\begin{array}{c}\text { Pulse location in } \\
\text { record }\end{array}$ & + & 0 & + & + & + \\
$\begin{array}{c}\text { Reflections in } \\
\text { signal }\end{array}$ & + & + & + & - & + \\
$\begin{array}{c}\text { Location } \\
\text { accuracy }\end{array}$ & + & + & + & - & $-/+^{\mathrm{b}}$ \\
\hline a) by incorporating channel's impulse response \\
b) if load impedances are known accurately
\end{tabular}

and characteristic cable impedance can be measured or estimated. If the load impedance is unknown, or if there are other locations in the cable circuit where the phase changes suddenly the accuracy of the phase method is poor.

\section{APPENDIX}

The major disadvantage of the phase method is that a phase shift in the transmission coefficient from cable to load impedance introduces a location error. This error can be corrected using equation (18) provided that the transmission coefficient is known accurately. The transmission coefficient is calculated using the characteristic cable impedance $Z_{c}$ and load impedance $Z_{\text {load }}$. Unfortunately, estimates of these quantities have limited accuracy. The sensitivity of the location accuracy to errors in the estimation of $Z_{c}$ and $Z_{\text {load }}$ can be derived as follows. Assuming that $Z_{c}$ is real and frequency-independent the phase of the transmission coefficient is given by:

$$
\angle T(\omega)=-\arctan \left(\frac{\sin \left(\alpha_{\text {load }}(\omega)\right) \cdot\left|Z_{\text {load }}(\omega)\right|}{Z_{c}+\cos \left(\alpha_{\text {load }}(\omega)\right) \cdot\left|Z_{\text {load }}(\omega)\right|}\right)
$$

with $\alpha_{\text {load }}$ the phase of $Z_{\text {load. }}$. In order to estimate the error due to the limited knowledge of $\alpha_{\text {load }}, Z_{\text {load }}$ and $Z_{c}$ we analyze the derivatives of (21) with respect to these parameters. The absolute value of the derivative of this equation to $\alpha_{\text {load }}$ is always less than one. The absolute value of the derivative of this equation to either $\left|Z_{\text {load }}\right|$ or $Z_{c}$ is less than 0.1 for practical values $\left(-90^{\circ} \leq \alpha_{\text {load }} \leq 90^{\circ}\right.$, $2 \leq\left|Z_{\text {load }}\right| \leq 200$, and $10 \leq Z_{c} \leq 30$ ). The next step is to use equation (14) to convert the phase to a time shift $t_{e}$ introduced at the transmission from cable to load impedance. The derivative of this time shift to $\angle T$ is:

$$
\frac{\partial t_{e}}{\partial\left(\angle T\left(\omega_{c}\right)\right)}=\frac{-1}{\omega_{c}}
$$

with $t_{e}$ the time shift/error introduced at the transmission from cable to load impedance. This indicates that $\omega_{c}$ should be chosen as high as the signal-to-noise ratio permits. The last step is to convert the time shifts at both ends $\left(t_{e l}\right.$ and $\left.t_{e 2}\right)$ to a location error. Assuming the 
PD location procedure described in section 4 this error is given by:

$e_{P D}=v_{p} t_{c} \frac{t_{e 1}-t_{e 2}-(x-1 / 2)\left(t_{e 1}+t_{e 2}\right)}{2 t_{c}+t_{e 1}+t_{e 2}}$

where $v_{p}$ the propagation velocity, $t_{c}$ the total propagation time of the cable, $t_{e l} / t_{e 2}$ the time shift introduced due to the phase shift in $T$ at respectively the near end and the far end, and $x$ the PD origin in fraction of the cable length. Normally, for cable systems $t_{c}>>$ $\left(t_{e l}+t_{e 2}\right)$. Using this assumption and calculating the derivatives to $t_{e l}$ and $t_{e 2}$ yields:

$$
\frac{\partial e_{P D}}{\partial t_{e 1}}=1 / 2 v_{p}[1-(x-1 / 2)] \text { and } \frac{\partial e_{P D}}{\partial t_{e 2}}=1 / 2 v_{p}[-1-(x-1 / 2)]
$$

Since $0 \leq x \leq 1$ the maximum of equation (24) is $3 / 4 v_{p}$. The result of an inaccurate estimation of $Z_{\text {load }}$ can now be estimated according:

$$
\Delta\left(e_{P D}\right)=\frac{\partial e_{P D}}{\partial t_{e}} \frac{\partial t_{e}}{\partial(\angle T(\omega))} \Delta(\angle T(\omega))
$$

If, for example, the estimation in the phase of $Z_{\text {load }}$ is $10^{\circ}$, the error in $\angle T$ is less than $10^{\circ}$. If $\omega_{c} / 2 \pi$ is $2 \mathrm{MHz}$ the error in $t_{e}$ is less than $14 \mathrm{~ns}$. Assuming $v_{p}=165 \mathrm{~m} / \mu$ s yields a location error less than $1.7 \mathrm{~m}$

\section{ACKNOWLEDGMENT}

The authors wish to thank KEMA Nederland B.V. and the Dutch utilities N.V. Continuon Netbeheer, ENECO Netbeheer B.V. and Essent Netwerk B.V. for supporting this research.

\section{REFERENCES}

[1] F. Steennis, P. van der Wielen, B. Kaptein, D. Harmsen, M. Kruithof and A. Postma, "Permanent On-line Monitoring of MV Power Cables Based on Partial Discharge Detection and Localisation - an Update", $7^{\text {th }}$ Int. Conf. Insul. Power Cables (Jicable), paper No. A 4.1, 2007.

[2] H. Zhang, T.R. Blackburn, B.T. Phung and D. Sen, "A Novel Wavelet Transform Technique for On-line Partial Discharge Measurements Part 1: WT De-noising Algorithm", IEEE Trans. Dielectr. Electr. Insul., Vol. 14, pp. 3-14, 2007.

[3] Y. Zhou, Y. Qin and P. Chappell, "Cost-Effective On-line Partial Discharge Measurements for Cables", IEEE Electr. Insul. Mag., Vol. 22, No. 2, pp. 31-38, 2006.

[4] M. Michel, "Innovative asset management and targeted investments using on-line partial discharge monitoring \& mapping techniques", Proc. $19^{\text {th }}$ Int. Conf. Electr. Distr. (CIRED), paper No. 0551, 2007.

[5] F.H. Kreuger, M.G. Wezelenburg, A.G. Wiemer and W.A. Sonneveld, "Partial Discharge Part XVIII: Errors in the Location of Partial Discharges in High Voltage Solid Dielectric Cables", IEEE Electr. Insul. Mag., Vol. 9, No. 6, 1993.

[6] G. Kitagawa and H. Akaike, "A procedure for the modeling of nonstationary time series", Ann. Inst. Stat. Math., Vol. 30, pp. 351-363, 1978.

[7] R. Sleeman and T. van Eck, "Robust automatic p-phase picking: an on-line implementation in the analysis of broadband seismogram recordings", Phys. Earth Planet. Inter., Vol. 113, pp. 265-275, 1999.

[8] H. Zhang, C. Thurber, and C. Rowe, "Automatic p-wave arrival detection and picking with multiscale wavelet analysis for single-component recordings", Bull. Seism. Soc. Am., Vol. 93, pp. 1904-1912, 2003.

[9] J.H. Kurz, C.U. Grosse, and H.-W. Reinhardt, "Strategies for reliable automatic onset time picking of acoustic emissions and of ultrasound signals in concrete", Ultrasonics, Vol. 43, pp. 538-546, 2005.

[10] C. Herold, T. Leibfried, S. Markalous, and I. Quint, "Algorithms for automated arrival time estimation of partial discharge signals in power cables", Proc. Int. Symp. High Volt. Eng. (ISH), paper No. T7-332, 2007.

[11] D. Gabor, "Theory of communication", J. IEE Pt. III: Radio Commun. Eng., Vol. 93, pp. 429-457, 1946.
[12] M. Mashikian, "Partial discharge location as a diagnostic tool for power cables", IEEE Power Eng. Soc. Winter Meeting, pp. 1604-1608, 2000.

[13] P.C.J.M. van der Wielen, On-line Detection and Location of Partial Discharges in Medium-voltage Power Cable, Ph.D. dissertation, Eindhoven University of Technology, Eindhoven, The Netherlands, 2005. Available: http://www.tue.nl/

[14] B. Iglewicz and D.C. Hoaglin, How to detect and handle outliers. Milwaukee, USA: ASQ Quality Press, 1993.

[15] P.A.A.F. Wouters, "Online calibration of high-frequency partial discharge signals in three-phase belted power cables", IEE Proc. Sci. Meas. Technol., Vol. 152, pp. 79-86, 2005.

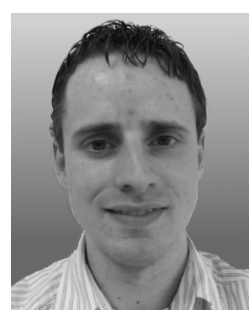

Paul Wagenaars (S'06) was born in Schaijk, The Netherlands in 1981. He received the M.Sc. degree in electrical engineering from the Eindhoven University of Technology, Eindhoven, The Netherlands in 2004. After his graduation he worked at KEMA on advanced calculation techniques for the current carrying capacity of power cables. Since 2006 he has been pursuing the Ph.D. degree. His current research mainly deals with online partial discharge monitoring of medium-voltage cable systems.

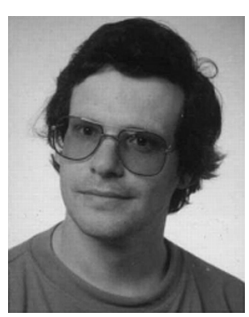

Peter A.A.F. Wouters was born in Eindhoven, The Netherlands, on 9 June 1957. He studied physics at the Utrecht University (UU), Utrecht, The Netherlands, until 1984, from which he received the $\mathrm{Ph} . \mathrm{D}$. degree for a study on elementary electronic transitions between metal surfaces and low energetic (multiple) charged ions in 1989. In 1990, he joined the Electrical Power Systems (EPS) group at the Technical University of Eindhoven, Eindhoven, The Netherlands, as Research Associate. His research interests include partial discharge techniques, vacuum insulation, and LF electromagnetic field screening. Currently, he is an Assistant Professor in the field of diagnostic techniques in high-voltage systems.

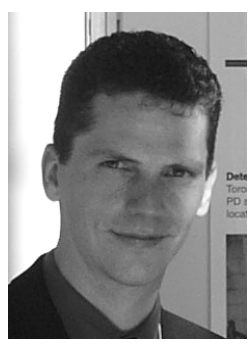

Peter C.J.M. van der Wielen (M'93) was born in Hulst, the Netherlands, on 6 March 1973. He studied electrical engineering at the Eindhoven University of Technology (EUT), where he was also a member of the board and many committees of the IEEE Student Branch Eindhoven. He received the M.Sc. degree in 2000. After that he started doing research on power cable diagnostics at both the Electrical Power Systems group at this university and KEMA, Transmission and Distribution Testing Services, in the Netherlands. In 2005 he received the Ph.D. degree for his study on on-line detection and location of PDs in medium voltage power cables. Since then he works as a consultant/specialist on power cables at KEMA. Subjects in the field of work are: power cable diagnostics, power cable consultancy in general, asset management, development of new measuring techniques \& diagnostic methods and failure analysis. Furthermore, he is one of the lecturers of the KEMA Power Cable Courses.

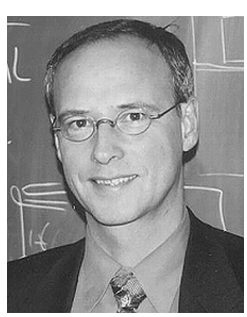

E. Fred Steennis joined KEMA, Arnhem, The Netherlands, in 1982, after his education at the Eindhoven University of Technology, Eindhoven, The Netherlands, where he studied degradation mechanisms in energy cables for the Dutch utilities. It was on this subject that he received the doctor's thesis from the Technical University in Delft, Delft, The Netherlands, in 1989. In and outside the Netherlands, he is a consultant on energy cables and is the author and a teacher of the KEMA course on Power Cables. At KEMA, he is currently senior consultant. Since 1999, he has also been a parttime Professor at the Technical University in Eindhoven, where he teaches and studies diagnostics for power cables. Prof. Dr. Ir. Steennis received the Hidde Nijland award for his contributions in 1991. After that, his experience on degradation mechanisms and related test methods both in the field and the laboratory was further enhanced. Based on this expertise, he became the Dutch representative with the Cigré Study Committee on High-Voltage Cables. He is also a member of various international Working Groups. 